Mode decomposition and Lagrangian structures of the flow dynamics in orbitally shaken bioreactors

Weheliye Hashi Weheliye, Neil Cagney, Gregorio Rodriguez, Martina Micheletti, and Andrea Ducci

Citation: Physics of Fluids 30, 033603 (2018); doi: 10.1063/1.5016305

View online: https://doi.org/10.1063/1.5016305

View Table of Contents: http://aip.scitation.org/toc/phf/30/3

Published by the American Institute of Physics

Articles you may be interested in

Dynamic metasurface lens based on MEMS technology

APL Photonics 3, 021302 (2018); 10.1063/1.5018865

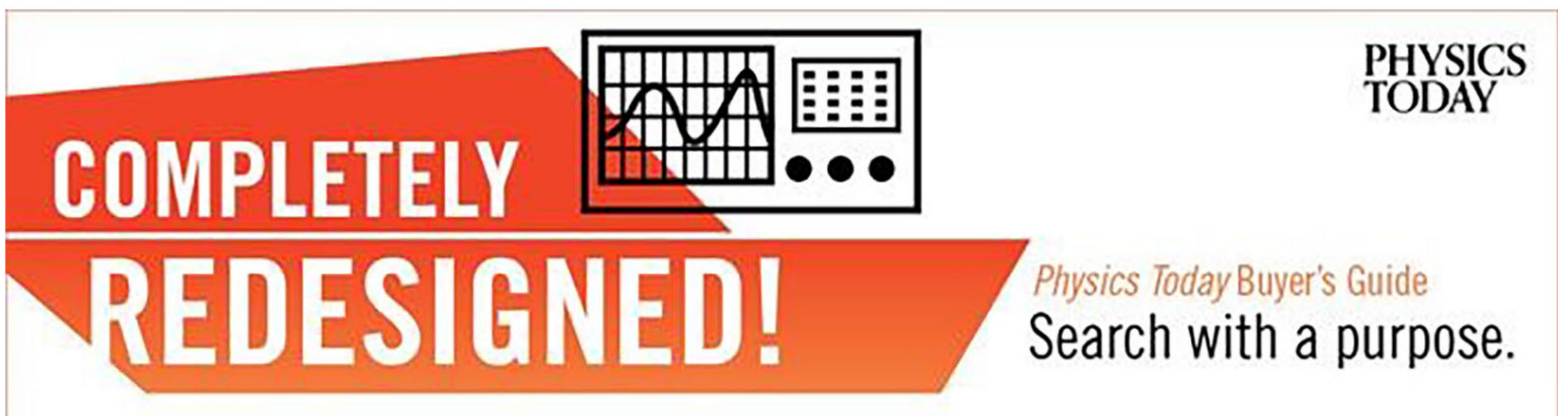




\title{
Mode decomposition and Lagrangian structures of the flow dynamics in orbitally shaken bioreactors
}

\author{
Weheliye Hashi Weheliye, ${ }^{1}$ Neil Cagney, ${ }^{2}$ Gregorio Rodriguez, ${ }^{3}$ Martina Micheletti, ${ }^{3}$ \\ and Andrea Ducci ${ }^{2, a)}$ \\ ${ }^{1}$ Chemical Engineering Department, University College London, Torrington Place, London WC1E 7JE, \\ United Kingdom \\ ${ }^{2}$ Mechanical Engineering Department, University College London, Torrington Place, London WC1E 7JE, \\ United Kingdom \\ ${ }^{3}$ Biochemical Engineering Department, University College London, Torrington Place, London WC1E 7JE, \\ United Kingdom
}

(Received 17 November 2017; accepted 7 February 2018; published online 13 March 2018)

\begin{abstract}
In this study, two mode decomposition techniques were applied and compared to assess the flow dynamics in an orbital shaken bioreactor (OSB) of cylindrical geometry and flat bottom: proper orthogonal decomposition and dynamic mode decomposition. Particle Image Velocimetry (PIV) experiments were carried out for different operating conditions including fluid height, $h$, and shaker rotational speed, $N$. A detailed flow analysis is provided for conditions when the fluid and vessel motions are in-phase $(F r=0.23)$ and out-of-phase $(F r=0.47)$. PIV measurements in vertical and horizontal planes were combined to reconstruct low order models of the full 3D flow and to determine its Finite-Time Lyapunov Exponent (FTLE) within OSBs. The combined results from the mode decomposition and the FTLE fields provide a useful insight into the flow dynamics and Lagrangian coherent structures in OSBs and offer a valuable tool to optimise bioprocess design in terms of mixing and cell suspension. Published by AIP Publishing. https://doi.org/10.1063/1.5016305
\end{abstract}

\section{INTRODUCTION}

Orbitally shaken bioreactors (OSBs) are extensively used in the early stages of bioprocess development and screening, where 24-, 48-, and 96-well plates provide an effective microscale platform to assess bioprocess performances in parallel ${ }^{1,2}$ (fermentation, enzyme bioconversion, and subsequent recovery steps). These come in different geometries either with a circular or a square cross section, while shaken flasks, either baffled or un-baffled, are most commonly used for larger volumes, $250-2500 \mathrm{ml}$. Shaken technology is often preferred to other agitation/mixing mechanisms for its low shear stresses and well-defined free surface for oxygen transfer. At production scale, stirred tank reactors are generally used, but in recent years, large-scale single used shaken bioreactors up to 10001 have been developed to provide a unique type of reactor at multiple scales. ${ }^{3,4}$ This can drastically simplify scaling up/down methodologies and provide consistent flow, mixing, and oxygen transfer dynamics in upstream bioprocessing. Flow and mixing related parameters of paramount importance for cell culture growth and scaling are oxygen transfer rate (proportional to the free surface interfacial area if a sparger is not present), energy dissipation ${ }^{5}$ and shear stresses, nutrients and $\mathrm{pH}$ distribution, ${ }^{6}$ and quality of cell suspension.

The first work to provide a visualisation of the flow and mixing in an orbitally shaken cylinder was that of Gardner and Tatterson, ${ }^{7}$ where the mixing characteristics were studied for varying operating conditions, including speed and viscosity.

\footnotetext{
a) Author to whom correspondence should be addressed: a.ducci@ucl.ac.uk
}

Since then most of the engineering characterization was done in terms of power consumption and oxygen transfer in shaken flasks, ${ }^{8,9}$ where distinction between "in-phase" and "out-ofphase flow conditions" was introduced. Numerical simulations of the flow in Erlenmeyer flasks and in micro-wells (24 and 96 plates) for fixed orbital diameter and reactor dimension were carried out by Zhang et al. ${ }^{10}$ and Zhang et al. ${ }^{1}$ respectively, while Kim and Kizito ${ }^{11}$ investigated the flow in an orbitally shaken cylindrical container for fluids of increasing viscosity by means of both simulations and flow visualisations. Detailed analyses of mean flow and turbulence characteristics in shaken cylinders with flat and conical bottoms for a large range of operating conditions, including fluid height, $h$, rotational speed, $N$, orbital diameter, $d_{o}$, vessel diameter, $d_{i}$, and fluid viscosity, $v$, are provided in Weheliye, Yianneskis, and Ducci, ${ }^{12}$ Ducci and Weheliye, ${ }^{13}$ and Rodriguez et al. ${ }^{14}$ These studies showed that the dynamics in OSBs are controlled by the Froude number, $F r=\frac{2 \pi^{2} N^{2} d_{o}}{g}$, and there is a critical Froude number, $F r_{c r}$, above which the flow transitions from being in phase with the orbital motion to out of phase. The critical Froude number is given by the empirical equation,

$$
F r_{c r}=\left\{\begin{array}{lll}
\frac{1}{a_{o w}}\left(\frac{h}{d_{i}}\right)\left(\frac{d_{o}}{d_{i}}\right)^{0.5} & \text { if } & \frac{h}{d_{i}}<\left(\frac{d_{o}}{d_{i}}\right)^{0.5} \\
\frac{1}{a_{o w}}\left(\frac{d_{o}}{d_{i}}\right) \frac{1}{a_{o w}} & \text { if } & \frac{h}{d_{i}}>\left(\frac{d_{o}}{d_{i}}\right)^{0.5},
\end{array}\right.
$$

where $a_{o w}$ is a non-dimensional coefficient dependent on the fluid viscosity (i.e., 1.4 for water) and $F r_{d_{i}}$ and $F r_{d_{o}}$ is the Froude number based either on the cylinder diameter, $d_{i}$, or orbital diameter, $d_{o}$.

Analytical solutions of the flow in orbiting cultures based on Stokes' second problem were compared to PIV and CFD 
results by Thomas et al., ${ }^{15}$ while potential flow functions were derived by Reclari et al. ${ }^{16}$ and Bouvard, Herreman, and Moisy ${ }^{17}$ and validated with PIV measurements. Similarly a potential sloshing model of the free surface was formulated and compared against free surface wave measurements by Reclari et al. ${ }^{16}$ who identified the presence of different modal responses inducing different flow regimes in a shaken cylindrical container, while Discacciati et al. ${ }^{18}$ developed a pressure correction method for CFD simulations, to best capture the free surface deformation and assess the shear stress levels for a highly viscous fluid. The flow scaling law of Weheliye, Yianneskis, and Ducci ${ }^{12}$ was successfully applied to the mixing time experiments of Rodriguez et al. ${ }^{19}$ and Rodriguez et al. ${ }^{20}$ obtained by means of an acid-base colorisation technique in shaken bioreactors of cylindrical geometry. Rodriguez et al. ${ }^{20}$ compared their data to those obtained by Tissot et al. ${ }^{21}$ for very different operating conditions $\left(d_{o}, h\right)$ and bioreactor sizes $\left(d_{i}\right)$ and found out that the two sets of data scaled well when the mixing number was plotted against the ratio of $F r / F r_{c r}$ and achieved a constant value after flow transition occurred $\left(F r>F r_{c r}\right)$. Similarly this Froude number ratio was also found to be an effective scaling tool to determine the minimum agitation speed for microcarrier suspension. ${ }^{22,23}$ These are small porous spheres, of the order of $100 \mu \mathrm{m}$, which are used to grow adherent cells, such as stem cells, in a 3D bioreactor environment (i.e., suspended over the entire fill volume). The two-phase PIV experiments of Pieralisi et al. ${ }^{23}$ showed that the flow characteristics in the presence of microcarriers are very close to those of a single phase system (i.e., small slip velocity).

In the current work mode decomposition techniques, such as POD and DMD, which have been extensively applied in the literature to stirred tank reactors, ${ }^{24-26}$ are for the first time applied to orbitally shaken systems, while Finite-Time Lyaponuv Exponent (FTLE) distributions were obtained from the PIV data and used to uncover Lagrangian coherent structures associated in OSBs. The objective of this study is to improve the understanding of the flow and mixing dynamics in shaken systems by means of modal decomposition, with the long-term vision to develop an alternative, effective approach to identify analogies between large scale flow structures of stirred and shaken reactors, therefore bridging scaling gaps between the two technologies.

The remainder of the paper is structured as follows: Sec. II A provides a brief description of the experimental apparatus and the post-processing techniques. The results are discussed in Sec. III, which illustrates the four main decomposition modes for low and high $F r$, while the Finite-Time Lyaponuv Exponent (FTLE) fields provide an insightful visualization of the large-scale Lagrangian coherent structures. The main conclusions drawn from this study are summarized in Sec. IV.

\section{EXPERIMENTAL APPARATUS AND POST-PROCESSING TECHNIQUES}

\section{A. Set-up}

Two-dimensional PIV was used to measure the velocity fields and to obtain modal decompositions and Lagrangian structures in an orbitally shaken bioreactor. The 2D-PIV system employed comprised of a continuous diode laser, a mirror, an intensified high-speed camera (Dantec Dynamics), and a cylindrical bioreactor rig (inner diameter $d_{i}=100 \mathrm{~mm}$ ), all of which were rigidly mounted on a shaker table (Lab LS-X Kühner). The cylindrical bioreactor was made of borosilicate glass (refractive index of 1.51), and to minimise refraction, it was encased in a square trough filled with water. To obtain optical access to the bioreactor from the bottom, the base of the cylinder was made of polished acrylic plastic.

Two sets of PIV measurements were carried out: (a) horizontal plane vector fields were obtained through time-resolved measurements to apply the mode decomposition techniques; and (b) vertical plane vector fields were obtained through phase-locked measurements because these are not affected by the free surface movement. ${ }^{12}$ Schematic diagrams of the PIV system setup for vertical and horizontal planes are provided in Figs. 1(a) and 1(b), respectively. For the phase-resolved measurements, a magnetic encoder was coupled to the Kühner shaker table to determine the angular position of the entire system (bioreactor and PIV) at any instant throughout its orbital trajectory. Phase-resolved measurements were averaged over 500 velocity fields and were obtained at different phase angles, $\phi$, by triggering the camera with a phase delay with respect to the encoder signal pulses. The origin of the phase angular coordinate, $\phi$, was set when the shaker tray reached its position furthest to the left along its clockwise circular orbit when seen from above. Time-resolved measurements included $2000 \mathrm{vec}-$ tor fields on horizontal planes, with image acquisition frame rates of $108 \mathrm{~Hz}$ and $390 \mathrm{~Hz}$ for $N=90 \mathrm{rpm}$ and $130 \mathrm{rpm}$, respectively.

The experiments presented in this work were carried out for an orbital diameter $d_{o}=50 \mathrm{~mm}$ and the working fluid was water $\left(v=10^{-6} \mathrm{~m}^{2} \mathrm{~s}^{-1}\right)$. Two fluid heights, $h=45 \mathrm{~mm}$ and $70 \mathrm{~mm}$, and shaker speeds, $N=90 \mathrm{rpm}$ and $130 \mathrm{rpm}$, were considered. Rhodamine fluorescent particles with diameter of 20-50 $\mu \mathrm{m}$ were used as tracers, and an orange filter with a cut-off wavelength of $570 \mathrm{~nm}$ was set on the camera mount to minimise reflections at the walls. An adaptive correlation

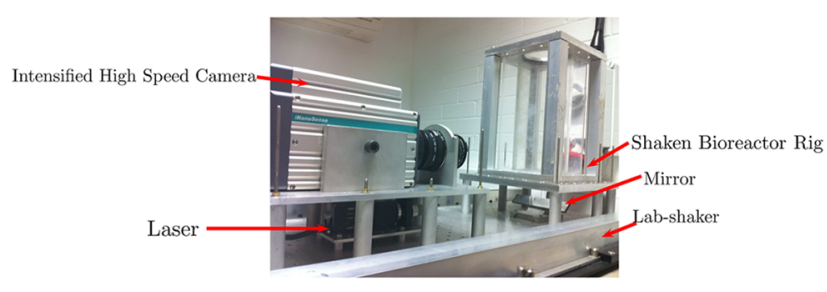

(a)

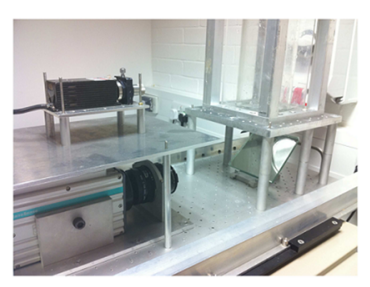

(b)

FIG. 1. Sketch of the PIV system set-ups used to measure the velocity field on (a) vertical and (b) horizontal planes. 
analysis of the full image was applied with an initial interrogation window of $256 \times 256$ pixels and a final window of $32 \times 32$ pixels with a $50 \%$ overlap. This resulted in a final spatial resolution of $1.7 \times 1.7 \mathrm{~mm}^{2}$. In the rest of the study, a cylindrical coordinate system, $r, \theta, z$ is employed with the origin positioned at the cylinder axis on the bioreactor base.

\section{B. POD and DMD approach}

The PIV velocity data obtained in this study were post-processed with two decomposition techniques, namely, Proper Orthogonal Decomposition (POD) and Dynamic Mode Decomposition (DMD). Both techniques are applied to the snapshot matrix, $X$, which is obtained by re-arranging the instantaneous velocity fields along different columns [see Eq. (2)],

$$
X=\left[\begin{array}{cccc}
u\left(x_{1}, t_{1}\right) & u\left(x_{1}, t_{2}\right) & \ldots \ldots & u\left(x_{1}, t_{N}\right) \\
u\left(x_{2}, t_{1}\right) & u\left(x_{2}, t_{2}\right) & \ldots \ldots \ldots & u\left(x_{2}, t_{N}\right) \\
\vdots & \vdots & \vdots & \vdots \\
\vdots & \vdots & \vdots & \vdots \\
u\left(x_{M}, t_{1}\right) & u\left(x_{M}, t_{2}\right) & \ldots \ldots & u\left(x_{M}, t_{N}\right)
\end{array}\right]
$$

The size of $X$ is $M \times N$, where $M$ and $N$ are the number of locations within the velocity field and the number of time instants collected, respectively. POD is a linear technique, based on temporal and spatial correlation analysis, that decomposes a set of signals into a modal base with modes ordered in terms of kinetic energy content (i.e., decreasing eigenvalues of the correlation tensor, $X^{T} \cdot X$; see Ref. 24). The first modes are the most energetic and associated with large scale structures, whereas the last modes are the least energetic and related to the small scale structures and turbulence. In Eq. (3), the POD analysis is applied to the fluctuating part of the velocity field, $\overrightarrow{\mathbf{u}}^{\prime}(\overrightarrow{\mathbf{x}}, t)$,

$$
\overrightarrow{\mathbf{u}}(\overrightarrow{\mathbf{x}}, t)=\overrightarrow{\mathbf{U}}(\overrightarrow{\mathbf{x}})+\overrightarrow{\mathbf{u}}^{\prime}(\overrightarrow{\mathbf{x}}, t)=\overrightarrow{\mathbf{U}}(\overrightarrow{\mathbf{x}})+\sum_{n=1}^{N_{s}} a_{n}(t) \vec{\Phi}_{n}(\overrightarrow{\mathbf{x}}),
$$

where $\overrightarrow{\mathbf{u}}(\overrightarrow{\mathbf{x}}, t)$ and $\overrightarrow{\mathbf{U}}(\overrightarrow{\mathbf{x}})$ are the instantaneous and mean velocity flow fields, respectively, whereas $\vec{\Phi}_{n}$ and $a_{n}$ are the spatial eigenfunction and the temporal coefficient associated to the $n$th mode, respectively. Depending on the flow of interest, a Low Order Model (LOM) can be utilised to reconstruct the large scale flow features and filter out turbulence and experimental error. The LOM would comprise a small number of modes that are characterised by large energy content. The LOM of Eq. (4) is based on the first four modes, which were found to be sufficient to fully describe the large scale flow features in stirred tank reactors for mixing applications, ${ }^{24-26}$

$$
\overrightarrow{\mathbf{u}}_{L O M}(\overrightarrow{\mathbf{x}}, \phi)=\overrightarrow{\mathbf{U}}(\overrightarrow{\mathbf{x}})+a_{1}(t) \vec{\Phi}_{1}(\overrightarrow{\mathbf{x}})+\cdots+a_{4}(t) \vec{\Phi}_{4}(\overrightarrow{\mathbf{x}}) .
$$

A detailed description of POD can be found in the work of Berkooz, Holmes, and Lumley. ${ }^{27}$

An alternative decomposition technique is DMD, which was first proposed by Schmid, ${ }^{28}$ and allows a better insight into the dynamics of a flow. DMD assumes a linear correlation between consecutive snapshots, as indicated in the following equation:

$$
X_{N}=A X_{N-1},
$$

where $X_{N-1}$ is a submatrix of $X$ including the first $N-1$ columns, while $X_{N}$ is comprised of columns $2-N$ of $X$. The dimension of the linear coefficient matrix, $A$, can be extremely large for PIV applications. The purpose of DMD is to determine a submatrix of $A, \widetilde{A}$, which contains the most energetic modes. The singular value decomposition of the snapshot submatrix $X_{N-1}$ is computed according to the following equation:

$$
X_{N-1}=U \Sigma V^{*},
$$

where matrices $U$ and $V$ contain the spatial structure and the temporal coefficient of the modes, respectively, while $\Sigma$, which is a diagonal matrix, provides the energy ranking of the modes. The asterisk in Eq. (6) denotes a matrix complex conjugate transpose. To reduce the computational cost and select a limited number of modes, $r$, where $r \ll M$ and $N, \widetilde{A}$ is estimated from Eq. (7) obtained by substituting the reduced singular value decomposition $\widetilde{U} \widetilde{\Sigma} \widetilde{V}^{*}$ into Eq. (5) and re-arranging

$$
\widetilde{A}=\widetilde{U}^{*} X \widetilde{V} \widetilde{\Sigma}^{-1},
$$

where $\widetilde{U}($ size $M \times r)$ and $\widetilde{V}(N-1 \times r)$ are comprised of the first $r$ columns of $U$ and $V$, respectively, and $\widetilde{\Sigma}$ is the $r$ submatrix of the diagonal matrix, $\Sigma$.

The eigenvalues, $\mu$, of $\widetilde{A}$ come in complex conjugate pairs and provide information on the temporal stability and dynamic characteristics of the $r$ selected modes. The real part of the eigenvalue represents the exponential increase/decay of a pair of modes, while its complex part provides their frequency of oscillation. The eigenvalues, $\mu$, are mapped logarithmically as $\lambda=\log (\mu) / \Delta t$ with $\Delta t$ being the temporal separation between two consecutive snapshots. The DMD spatial modes are estimated according to Eq. (8), where the reduced-matrix $\widetilde{U}$ is projected on $y_{i}$, which is the $i$ th eigenvector of the reduced linear coefficient matrix, $\widetilde{A}$,

$$
\Phi_{D M D i}=\widetilde{U} y_{i}
$$

A detail description of the DMD can be found in the works of Schmid $^{28}$ and Brunton et al. ${ }^{29}$

The main difference between POD and DMD is related to the fact that POD modes are ranked in terms of kinetic energy content, while DMD modes are associated with specific frequencies. This implies that energy associated with a specific frequency might be spread over more than one mode, when POD is applied. Similarly it is not possible to know a priori whether DMD modes associated with a certain frequency are the most energetic. Therefore the two techniques are complementary and both were used in this study because they can offer an effective tool to identify the most energetic structure and characterise their dynamic response.

\section{Finite-time Lyaponuv exponent (FTLE)}

Finite-Time Lyapunov Exponent (FTLE) fields represent the local rate of fluid stretching and are often used to approximate the Lagrangian Coherent Structures (LCS) ${ }^{30}$ and can be used to reveal the complex processes governing flows ${ }^{30}$ FTLE has been used extensively to study a wide range of flows and to decompose the flow into distinct regions. ${ }^{31,32}$ Despite the power of the FTLE approach for studying mixing, ${ }^{33}$ there has been relatively little or no experimental work investigating the 
Lagrangian dynamics in mixing vessels, or more specifically, in shaken bioreactors.

The estimation of Lagrangian properties such as FTLE typically begins by considering a uniformly spaced grid of massless tracers with positions, $\mathbf{x}_{0}$ at time $t_{0}$. These can be numerically advected forwards in time (or backwards, in some cases) through the measured velocity fields in order to find the pathlines for each tracer. The instantaneous position of these tracers is represented by the "flow map," $\varphi$, a tensor which depends on the initial tracer locations $\left(\mathbf{x}_{0}\right.$ and $\left.t_{0}\right)$ and varies with time.

The local rate of stretching is found by tracking the separation between initially neighbouring tracers. In practice, the maximum rate of stretching of a tracer is identified by finding the largest real eigenvalue, $\lambda_{\max }$, of the Cauchy-Green deformation tensor, which is given by

$$
C=(\nabla \varphi(\mathrm{x}))^{\top}(\nabla \varphi(\mathrm{x})) .
$$

The FTLE field represents the rate of exponential stretching of a fluid element and can thus be found as

$$
\sigma_{f}=\frac{1}{\left|t-t_{o}\right|} \log \sqrt{\lambda_{\max }}
$$

A local region of evaluation $\sigma_{f}$ indicates a region where the fluid will undergo rapid stretching over the period $t_{0}$ to $t$. In the current work, the FTLE field is evaluated for fluid elements originating in the horizontal plane $z / d_{i}=0.03$. The initial tracer grid has a spatial resolution twice that of the PIV measurements, i.e., the tracers have an initial separation of $0.85 \mathrm{~mm}$. The tracers were advected forward in time using a fourth order Runge-Kutta scheme, through the threedimensional velocity field which is composed of the low-order POD models in 12 horizontal planes $\left(z / d_{i}=0.015-0.165\right)$ and the phase-averaged measurements in the vertical planes.

For the high $\mathrm{Fr}=0.47$ case, the greater vertical oscillations in the free surface height meant that the vertical velocity fields could not be accurately measured above $z / d_{i}=0.1$, and many of the tracers advected out of the measurement in less than half a shaking cycle, before the FTLE fields could be fully resolved. Therefore, the FTLE data are presented only for the low $F r$ case.

\section{RESULTS}

\section{A. POD and DMD methods}

In this section, the 2D PIV measurements in the horizontal planes are first analyzed using POD. The percentage of kinetic energy associated with the first 10 modes for varying Froude number, $F r$, non-dimensional fluid height, $h / d_{i}$, and non-dimensional axial coordinate of the horizontal measurement plane, $z / d_{i}$, is presented in Figs. 2(a)-2(c). The results in Fig. 2 take into account only the fluctuating component, $\overrightarrow{\mathbf{u}}^{\prime}$, of Eq. (3), while the corresponding ensemble average kinetic energy has been removed before applying the POD analysis. For a fixed fluid height, $h / d_{i}=0.45$, and axial distance from the reactor bottom, $z / d_{i}=0.1$ [see Fig. 2(a)], the content of kinetic energy decreases when higher POD modes are considered, as expected. It can be seen that the first two modes contain nearly $96 \%$ of the total fluctuating kinetic energy for $\mathrm{Fr}=0.23$, while this decreases to $70 \%$ when the highest rotational speed is considered (i.e., $F r=0.47$ ). This is in agreement with the findings of Ducci and Weheliye ${ }^{13}$ who showed for $F r / F r_{c r}>1$ the outof-phase degree of the flow increases and a phase-resolved flow transition occurs, with some turbulence developing (this is present in the higher order modes). For the operating conditions, $h / d_{i}=0.45$ and $d_{o} / d_{i}=0.5$, of Fig. 2(a), the critical Froude number is $F r_{c r} \approx 0.23$, and as a result, the percentage energy content of higher modes, mainly 3 and 4 , increases. This is more pronounced for the highest Froude number considered, $F r=0.47$ (i.e., $F r / F r_{c r}>2$ ), where the phaseresolved flow transition has occurred and the contribution of the first two modes is only 7 times greater than those of modes 3 and 4.

Figure 2(b) shows the variation of the normalised eigenvalues, $\lambda_{1}$ and $\lambda_{2}$, with increasing $F r$ for two non-dimensional fluid heights, $h / d_{i}=0.45$ and 0.7 , at a fixed axial coordinate, $z / d_{i}=0.1$ (i.e., horizontal plane). It is interesting to point that $\lambda_{1}$ and $\lambda_{2}$ increase with $F r$ with a similar rate for both heights analyzed. The difference in magnitude is explained by considering that for the highest fluid height, $h / d_{i}=0.7$, the measurement plane is further away from the free surface (the flow driving mechanism) for the lowest height investigated, $h / d_{i}=0.45$. This behavior can be explained by considering the flow dynamics obtained by Weheliye, Yianneskis, and Ducci, ${ }^{12}$ who reported that the flow in the shaken reactor is characterised by a toroidal vortex beneath the free surface and a slow moving diffusion zone at the bottom of the reactor. As $\mathrm{Fr}$ is increased, the toroidal vortex extends toward and reaches the bottom of the reactor for $F r=F r_{c r}$. For a fluid height $h / d_{i}=0.45$, the corresponding critical Froude number is $F r_{c r} \approx 0.23$, while for $h / d_{i}=0.7$ is $F r_{c r} \approx 0.35$. Based on this, it can be concluded that for $h / d_{i}=0.45$, the plane $z / d_{i}=0.1$ is within the toroidal vortex region for the entire range of $\mathrm{Fr}$ investigated, while for $h / d_{i}=0.7$, the measurement plane is mostly within the diffusion zone.

This behavior is well reflected in Fig. 2(c) where the normalised eigenvalues obtained at four different axial coordinates $z / d_{i}=0.04-0.16$ are provided for $h / d_{i}=0.45$ and $F r=0.23\left(F r / F r_{c r} \approx 1\right)$. The normalised $\lambda_{i}$ is greater at the highest elevation considered, $z / d_{i}=0.16$, for the first two modes because the measurement plane is closer to the free surface, and $\lambda_{i}$ decreases as lower elevations are considered ( $18 \%$ decrease). The energy content at the bottom of the reactor, $z / d_{i}=0.04$, is relevant to the suspension of micro-carriers, and, for example, Pieralisi et al. ${ }^{23}$ have shown that suspension of GE Cytodex 3 micro-carriers occur for $F r \approx 1.1 F r_{c r}$. From this perspective, the intensity range of the first two eigenvalues shown in Fig. 2(c) has the potential to provide a kinetic energy benchmark, based on a single flow parameter, to assess the quality of suspension within the reactor.

The cyclic variation of the flow as the bioreactor progresses along its orbit was investigated by analyzing the phaseresolved temporal coefficients, $a_{n}$, of the four most energetic modes. The analysis was repeated for two Froude numbers for in-phase $[F r=0.23$, Fig. 3(a) $]$ and out-of-phase flow $[F r=0.47$, Fig. 3(b)]. For $F r=0.23$ [see Fig. 3(a)], modes 1 and 2 exhibit similar amplitudes, $\approx 3.5$, and are directly related to the orbital 


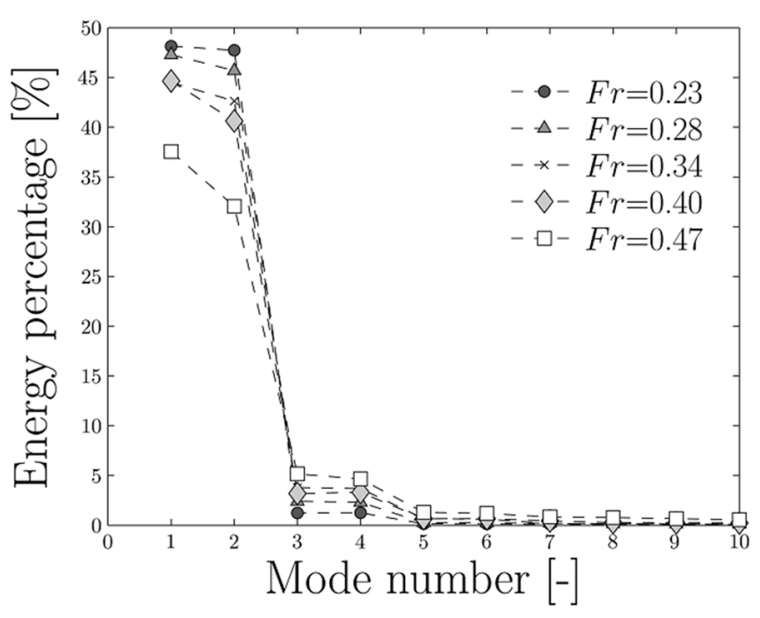

(a)

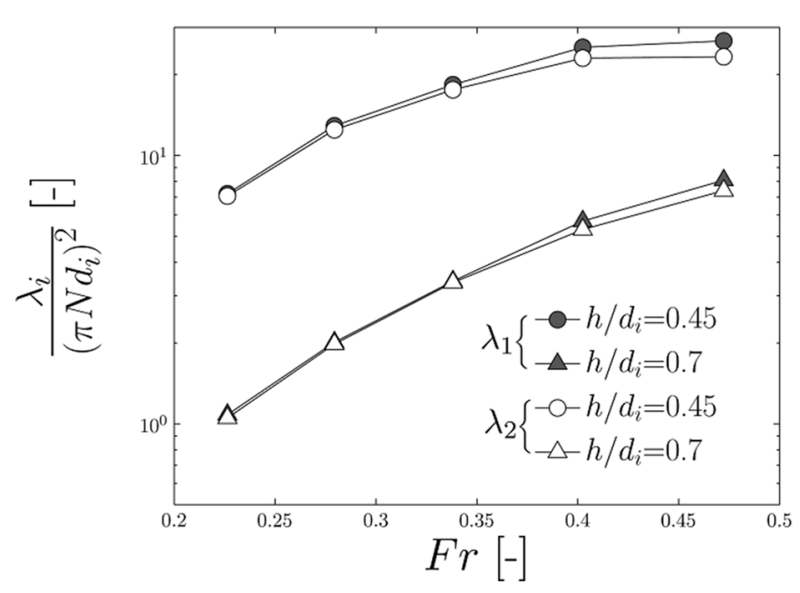

(b)

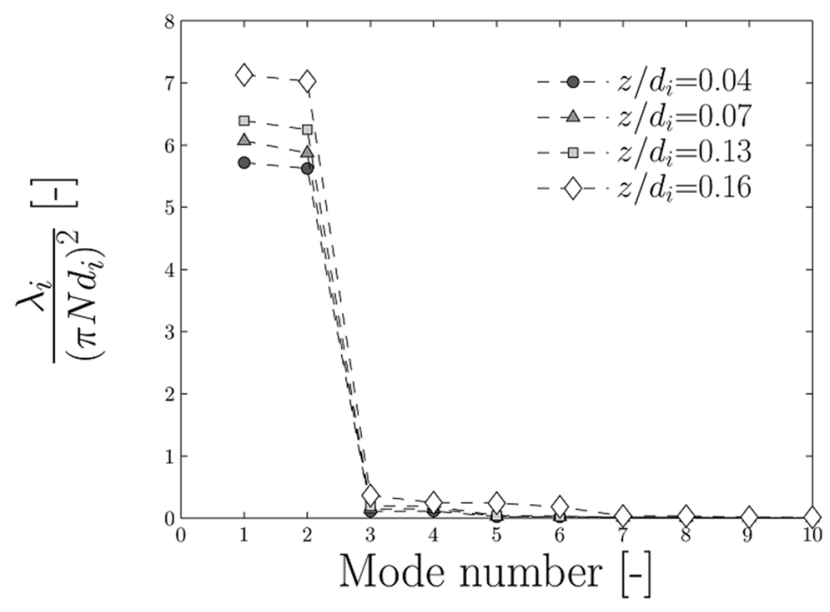

(c)

FIG. 2. Percentage of energy associated with each mode for increasing (a) Froude number, $F r\left(h / d_{i}=0.45\right.$ and $\left.z / d_{i}=0.1\right)$; (b) fluid non-dimensional height, $h / d_{i}\left(z / d_{i}=0.1\right)$; (c) axial coordinate of measurement plane, $z / d_{i}\left(h / d_{i}=0.45\right.$ and $\left.F r=0.23\right)$.

motion of the table with an oscillation frequency, $f=N$. A $90^{\circ}$ phase difference between the two coefficients is evident, which confirms the orthogonality of the first two temporal modes. Similarly, the coefficients $a_{3}$ and $a_{4}$ are orthogonal to each other with a $45^{\circ}$ phase lag between the two over a single orbital revolution. Their frequency is double of that displayed by modes 1 and 2, while their amplitudes is significantly smaller. Similar characteristics are observed in the temporal coefficients obtained for $F r=0.47$. However, in this case, the magnitude of $a_{3}$ and $a_{4}$ is significantly higher, nearly half of that exhibited by $a_{1}$ and $a_{2}$, and their contributions to the local flow cannot be discarded at this $F r$.

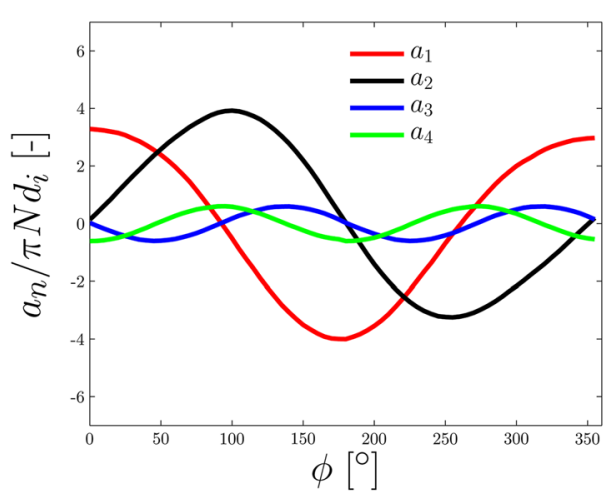

(a)

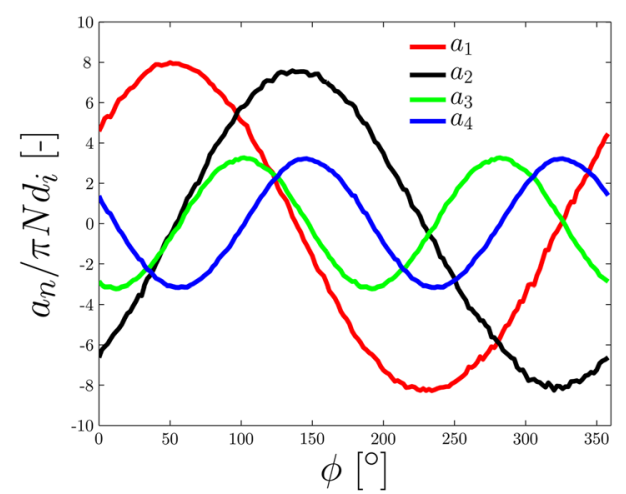

(b)
FIG. 3. Variation of the phase-resolved temporal coefficients $a_{1}, a_{2}, a_{3}$, and $a_{4}$ normalised with $\pi N d_{i}$ for $\left(h / d_{i}=0.45\right.$ and $z / d_{i}=0.1$ ): (a) $F r=0.23$; (b) $F r=$ 0.47 . 
An improved insight into the characteristic flow fields for the in-phase and out-phase conditions can be gained by plotting the spatial eigenfunctions, $\vec{\Phi}_{n}$, for the first four POD modes, $n=1-4$. The corresponding velocity vector fields for $F r=0.23$ and 0.47 are shown in Figs. 4 and 5, respectively. From Figs. 4(a) and 4(b), it is evident that the flow structures of modes 1 and 2 are offset by $90^{\circ}$ in the tangential direction. The flow is characterised by a strong radial stream which is at any time orthogonal to the centrifugal force induced by the orbital rotation. Two in-plane stagnation points, $180^{\circ}$ apart, can be distinguished, where the main flow stream diverges from and converges to. This characteristic flow pattern was consistently present for all the axial coordinates investigated and did not display any azimuthal angular offset for different $z / d_{i}$. In addition, modes 1 and 2 can be used to assess the degree of inphase and out-of-phase flow. From this point of view, Bouvard, Herreman, and Moisy ${ }^{17}$ determined the degree of out-of-phase flow by comparing the direction of the velocity at the centre of the reactor and the direction orthogonal to the centrifugal acceleration. According to this definition, for $F r=0.23$, the flow is always in-phase with the orbital motion of the bioreactor for any given phase angle. In fact from Fig. 3(a) at phase angle $\phi=0^{\circ}, a_{1}$ reaches maximum amplitude, while $a_{2}=0$,

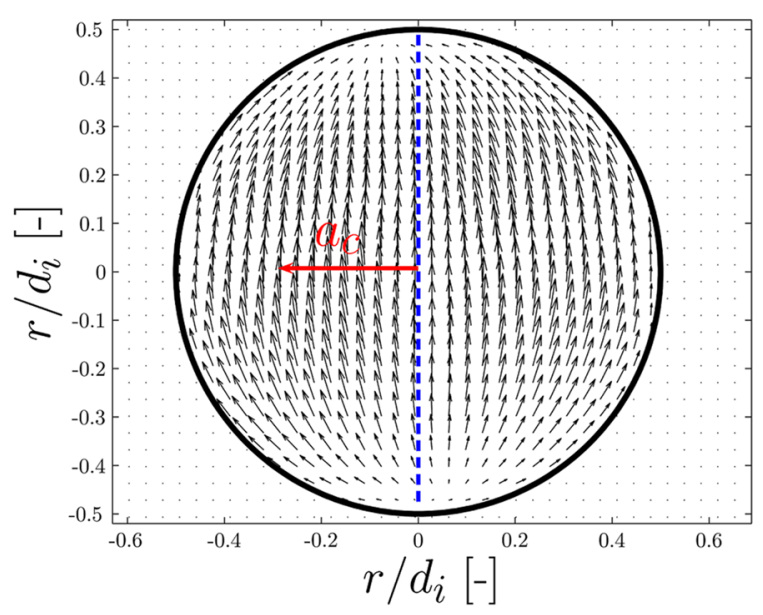

(a)

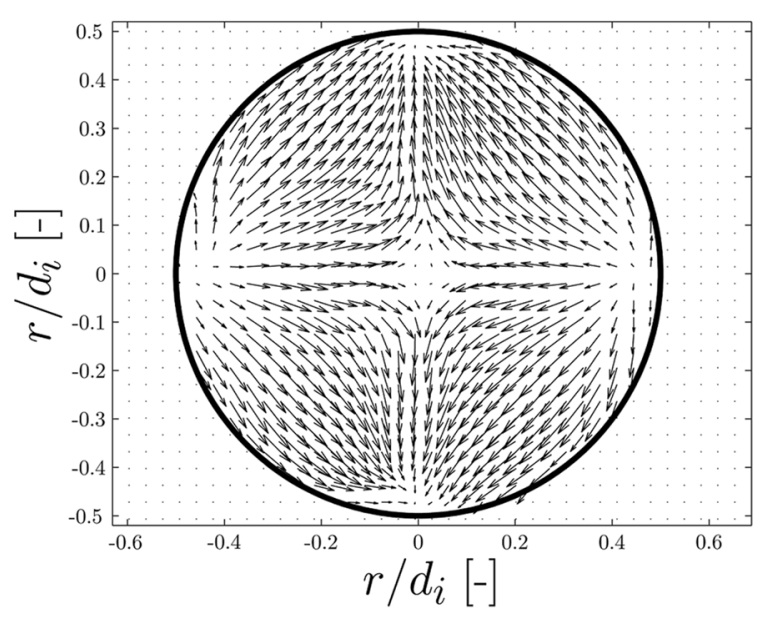

(c) therefore the flow field of mode 1, Fig. 4(a), is representative of that of the low order model generated by combining modes 1 and 2, at phase angle $\phi=0^{\circ}$. In Fig. 4(a), it is evident that the flow at the centre of the bioreactor is aligned with the blue dashed line orthogonal to the centrifugal acceleration, $a_{c}$ (red arrow), which at $\phi=0^{\circ}$ is oriented horizontally. Similarly, at $\phi=90^{\circ}, a_{2}$ reaches its maximum amplitude and $a_{1}=0$. This implies that the low order model of modes 1 and 2 is represented by Fig. 4(b). Also in this case the flow at the centre of the bioreactor is aligned with the direction orthogonal to the centrifugal acceleration [vertical in Fig. 4(b)]. The velocity fields of modes 3 and 4 shown in Figs. 4(c) and 4(d) are very different as they exhibit a hyperbolic flow pattern with a single in-plane stagnation region at the centre of the bioreactor. The velocity fields are shifted along the tangential direction by $45^{\circ}$.

Similarly to the low Froude number case, the flow fields of the first two modes for $\mathrm{Fr}=0.47(N=130 \mathrm{rpm})$ exhibit two in-plane stagnation points $180^{\circ}$ apart [see Figs. 5(a) and 5 (b)]. In this case, however, the main flow is forced to follow the bioreactor wall curvature, while its intensity in the core of the bioreactor is significantly reduced. The degree of out-ofphase flow can also be estimated at $F r=0.47$ from the low

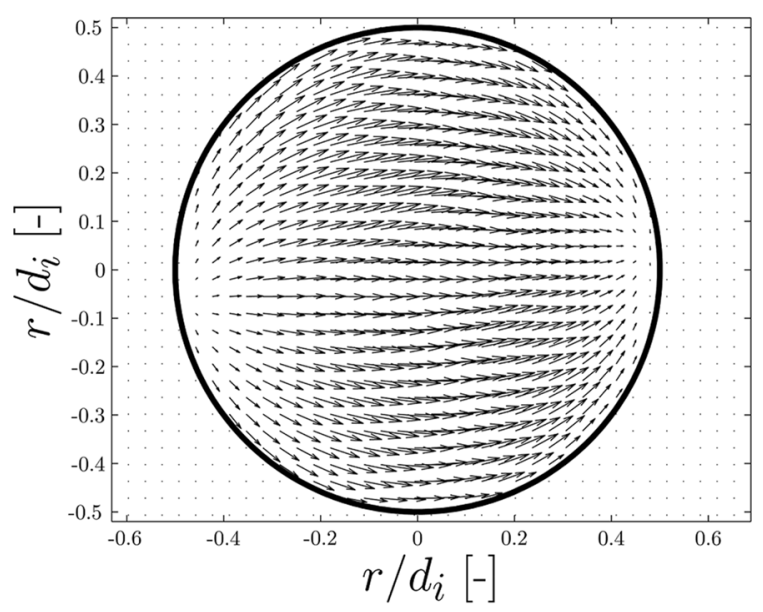

(b)

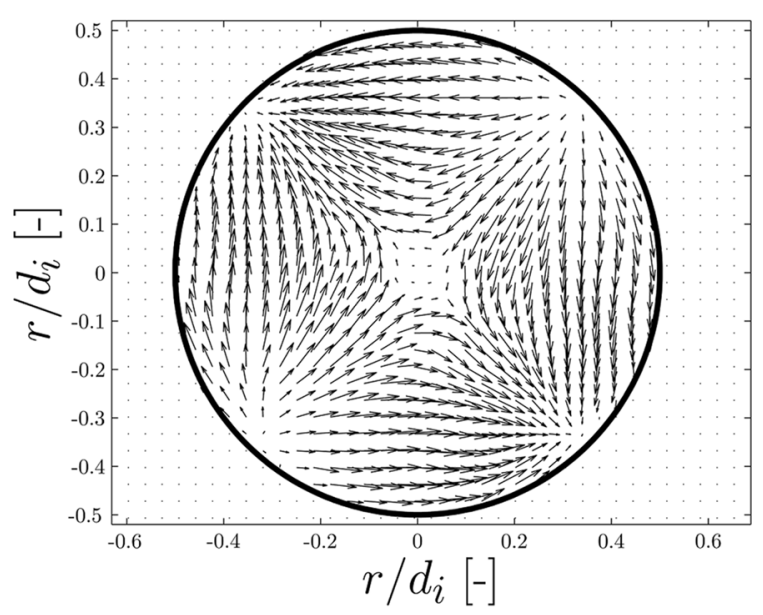

(d)

FIG. 4. Plot of the velocity fields of the first four modes for $F r=0.23\left(h / d_{i}=0.45\right.$ and $\left.z / d_{i}=0.1\right)$ : (a) mode 1 ; (b) mode 2 ; (c) mode 3 ; (d) mode 4 . 


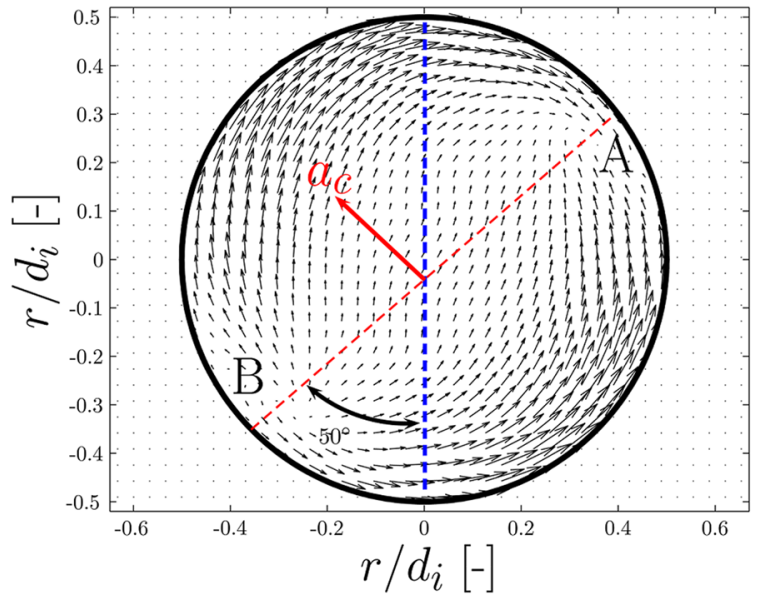

(a)

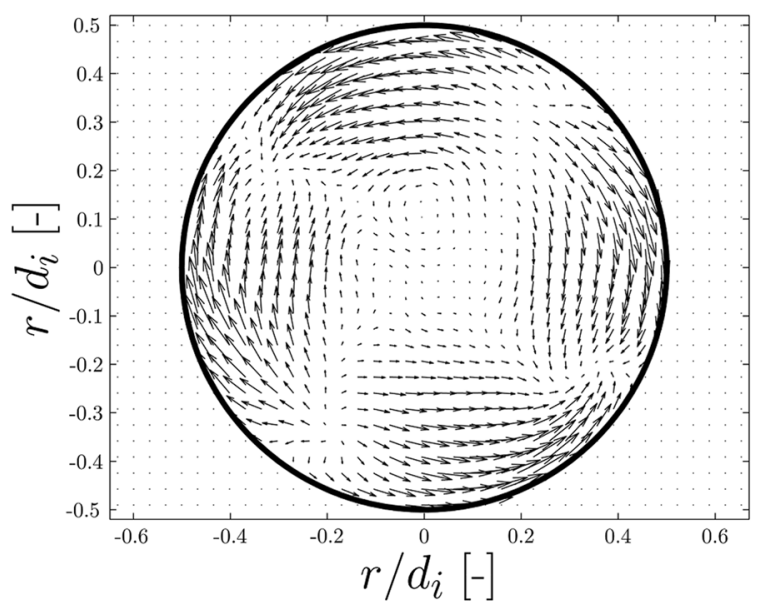

(c)

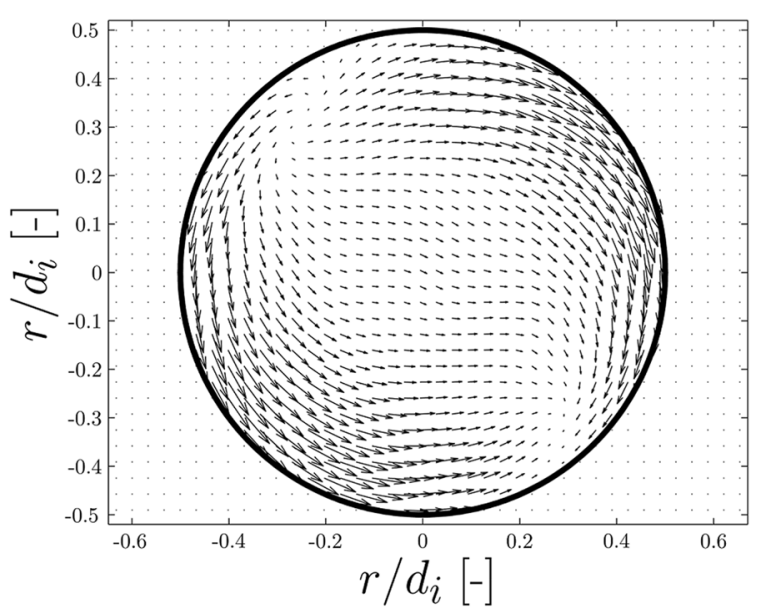

(b)

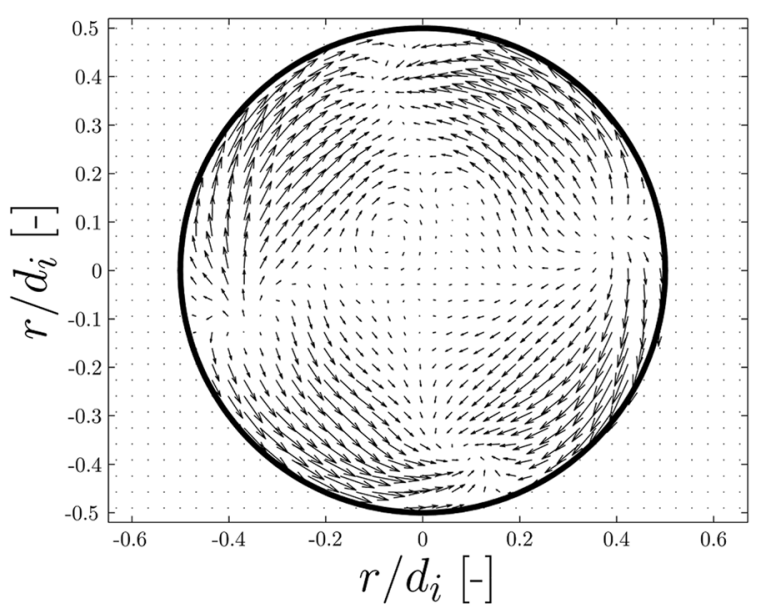

(d)

FIG. 5. Plot of the velocity fields of the first four modes for $F r=0.47\left(h / d_{i}=0.45\right.$ and $\left.z / d_{i}=0.1\right)$ : (a) mode 1 ; (b) mode 2 ; (c) mode 3 ; (d) mode 4 .

order model obtained from the combination of modes 1 and 2 . According to Fig. 3(b), $a_{1}$ reaches its maximum amplitude at $\phi=50^{\circ}$ and $a_{2}=0$. This means that the velocity field of mode 1, Fig. 5(a), is a good representation of the low order model of modes 1 and 2 at $\phi=50^{\circ}$. At this phase angle, the centrifugal acceleration is oriented as the red arrow, and the line orthogonal to the acceleration is denoted by the blue dashed line. It is evident that the flow at the centre, which points upward, is out of phase by approximately $50^{\circ}$. This analysis can be repeated for other $\phi$, and the phase difference remain unvaried. Therefore it can be concluded that modes 1 and 2 can be used to estimate the degree of out-of-phase flow at different $F r$.

The flow fields of modes 3 and 4 shown in Figs. 5(c) and 5(d) display two distinctive features. First, four small flow regions are observed close to the proximity of the walls of the bioreactor. These are separated by an angle of $90^{\circ}$, and the flow tangential direction is the same for regions on the opposite side of the bioreactor. Second, the area occupied by the near zero velocity in the core of the bioreactor is larger for $\mathrm{Fr}=0.47$ when compared with that for $F r=0.23$ (i.e., faster motion close to the wall). For $F r=0.47$, the flow fields of modes 3 and 4 recall the POD modes associated with the trailing vortices described in a stirred tank reactor for four-blade ${ }^{25}$ and six-blade ${ }^{24}$ impellers. In these cases, each trailing vortex was characterised by a pair of opposite flow streams, which indicates that the flow field shown in Figs. 5(c) and 5(d) is potentially analogous to that produced by a two-blade impeller. In order to assess the mixing performance of different modes, the rate of viscous dissipation of kinetic energy ${ }^{13}$ was estimated for each mode at different Froude number $F r$. For both $F r$, modes 1 and 2 exhibited higher dissipation close to the wall, while the centre was mainly characterised by negligible dissipation. This did not occur for modes 3 and 4 where non-zero velocity gradients are present in the bulk of the flow. Based on this analysis, it can be concluded that modes 3 and 4 improve mixing in the bulk of the flow.

A comparison was also made between the flow field reconstructed from modes 1 and 2 at both $\mathrm{Fr}$ and the flow field computed by Bouvard, Herreman, and Moisy ${ }^{17}$ from the potential function (inviscid flow assumption). It can be seen that at low $\mathrm{Fr}$ the velocity fields of modes 1 and 2 are very similar to that obtained from the potential function. However, when $\mathrm{Fr}$ is increased, the potential function fails to predict any degree of out-of-phase flow, which was reported to increase in the PIV measurements of Bouvard, Herreman, and Moisy, ${ }^{17}$ and the 
(a)

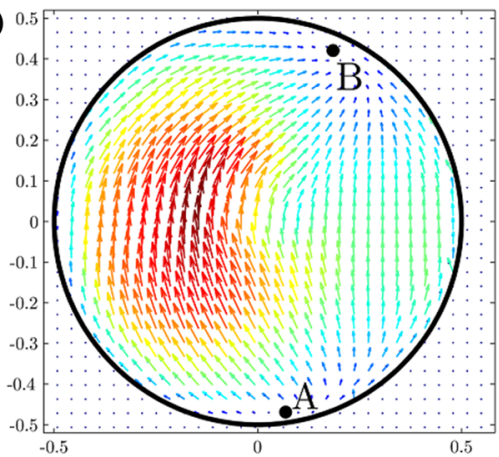

(c)

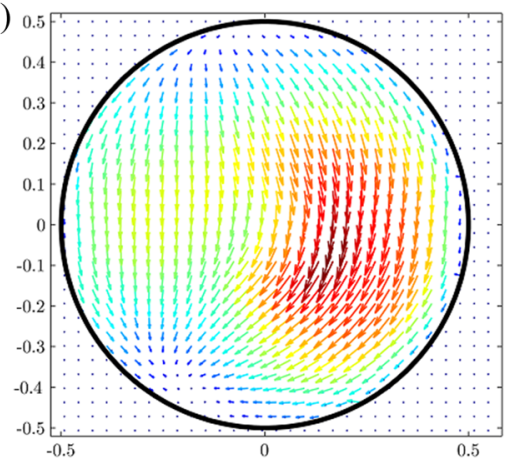

$0^{0.14} \quad$ (b)

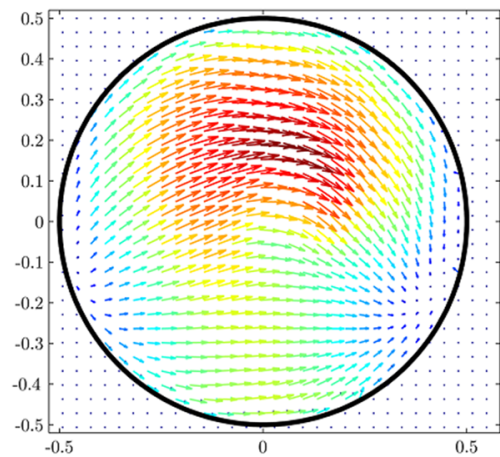

(d)

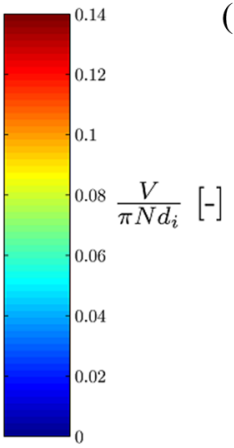

b)

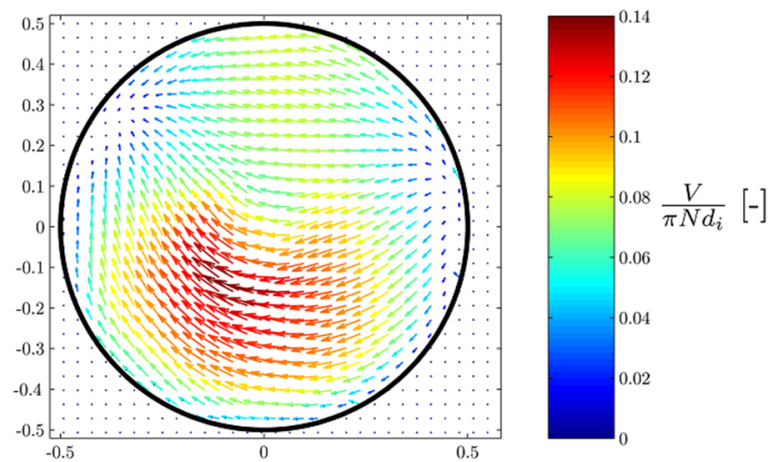

FIG. 6. Vector plots of the superimposed LOM for four values of phase angle, $\phi\left(F r=0.23, z / d_{i}=0.1\right)$ : (a) $\phi=0^{\circ}$; (b) $\phi=90^{\circ} ;$ (c) $\phi=180^{\circ}$; (d) $\phi=270^{\circ}$.

general shape of the flow field is different to that obtained in this study, where larger velocities are present near the wall.

Further understanding on the interaction between the different modes identified from the POD analysis can be gained from Figs. 6 and 7, where the velocity vector fields of the Low Order Model (LOM) obtained from Eq. (4) is shown at four phase angles, $\phi$, for $F r=0.23$ and 0.47 , respectively. For both $\mathrm{Fr}$, the same flow structure is consistently present throughout the orbital motion. It is interesting to point out that for $F r=0.23$, the entire flow is mainly in the direction of the shaker table movement. This behavior is determined by modes 1 and 2, where the main stream is always orthogonal (a)

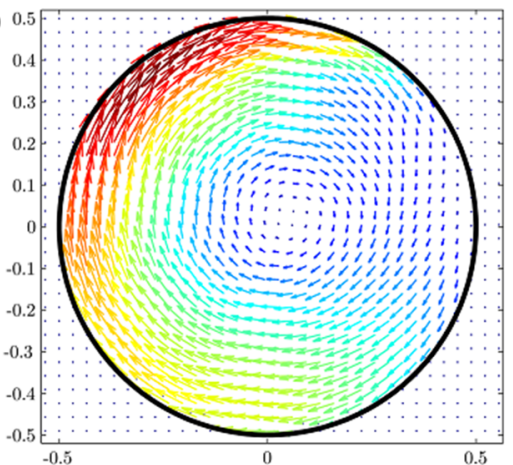

(c)

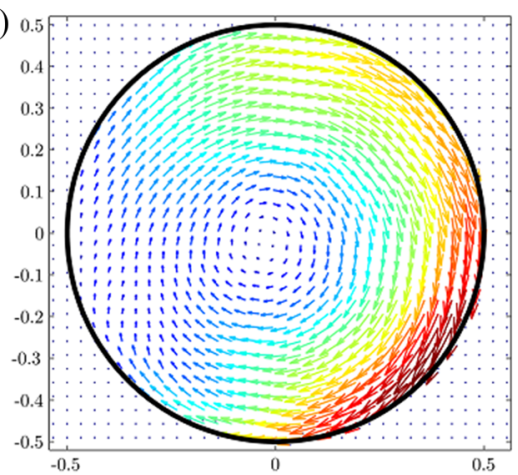

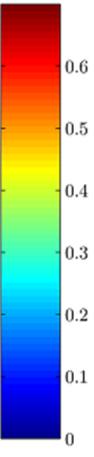

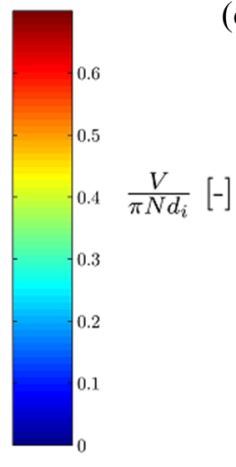

(b)

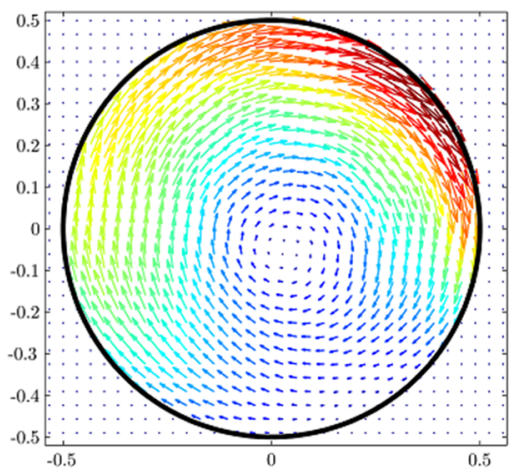

(d)

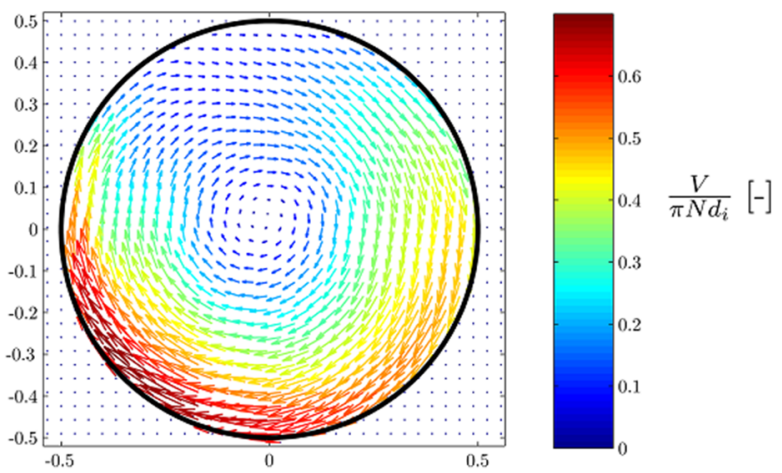

FIG. 7. Vector plots of the superimposed LOM for four values of phase angle, $\phi\left(F r=0.47, z / d_{i}=0.1\right)$ : (a) $\phi=0^{\circ} ;\left(\right.$ b) $\phi=90^{\circ} ;\left(\right.$ c) $\phi=180^{\circ} ;(d) \phi=270^{\circ}$. 
to the centrifugal acceleration of the bioreactor (large radial velocity at the centre), while the mean flow pattern (not shown here) is characterised by solid body rotation (mainly tangential velocity component, $u_{\theta}$ ). In the LOM velocity fields, the in-plane stagnations points [denoted as A and B in Fig. 6(a)] are approximately $130^{\circ}$ apart, nearly $50^{\circ}$ less than that exhibited by the corresponding velocity fields for modes 1 and 2 . This is determined by the intensity of the mean motion, as this angle decreases with increasing rotational speed (i.e., greater mean motion). These results are in agreement with the phaseresolved measurements of Weheliye, Yianneskis, and Ducci ${ }^{12}$ for in-phase flow.

The flow fields for $\mathrm{Fr}=0.47$ is characterized by a vortex with a vertical axis as shown in Figs. 7(a)-7(d). In this case, the opposite wall streams of modes 1 and 2 locally enhance and suppress the solid body rotation of the mean flow (not shown here), resulting in a faster flow along the wall furthest away from the centre of rotation. In this case, the flow field does not show an in-plane stagnation point because the intensity of the mean flow is always greater than the wall stream of modes 1 and 2 rotating in the opposite direction. The region at the centre is denoted by low flow intensity for all the modes and mean flow velocity fields, and this is reflected in the corresponding LOM velocity fields of Figs. 7(a)-7(d).

The 2D PIV horizontal data were also processed using the Dynamic Mode Decomposition (DMD) algorithm described in Sec. II B. Figure 8(a) shows the eigenvalue spectrum associated with the different modes for $F r=0.23$ and $F r=0.47$. The real part of the eigenvalue, $\lambda_{r}$, denotes the growth/decay of the mode, while the imaginary part $\lambda_{i}$ represents the frequency of the oscillation, $f=\lambda_{i} / 2 \pi$. It should be noted that the symbol size and colour of the eigenvalues in Fig. 8(a) are intended to separate large scale energetic structures (large symbols) from smaller-scale less energetic structures (small symbols). Distinction between high and low energy modes was done $a$ posteriori after comparison with the POD modes. For both $F r=0.23$ and 0.47 , there is an eigenvalue located at the origin $\left(\lambda_{r}=0, \lambda_{i}=0\right)$, which corresponds to the mean flow structure. For $\Phi_{D M D 1}$ and $\Phi_{D M D 3}$, at $F r=0.23$, the eigenvalues lie on the imaginary axis at $\lambda_{i}=9.421$ and $18.65 \mathrm{~s}^{-1}$, respectively, corresponding to frequencies of $f=1.5$ and $3 \mathrm{~Hz}$. This is in good agreement with the findings of the frequencies obtained for modes 1 and 3 from the POD for $F r=0.23$. In addition to this, the absence of exponential decay/growth $\left(\lambda_{r 1}=\lambda_{r 3} \approx 0\right)$ is observed for both DMD modes, which is expected for a forced oscillatory system like the shaken bioreactor. Similarly for $F r=0.47$, the frequency of mode $3\left(\lambda_{i}=27.06 \mathrm{~s}^{-1}\right.$, $f=4.3 \mathrm{~Hz})$ is twice of that of mode $1\left(\lambda_{i}=13.7 \mathrm{~s}^{-1}\right.$, $f=2.18 \mathrm{~Hz}$ ), which is in agreement with the temporal coefficients of the POD analysis [cf. Fig. 3(b)]. The other modes in Fig. 8(a) are denoted by a negative real part, $\lambda_{r}<5$, which implies that the amplitude of the slowest oscillations will decay of $95 \%$ within the first $0.6 \mathrm{~s}$ (cf. approximately the first cycle of the shaker table, $0.66 \mathrm{~s}$, for $F r=0.23$ ). Figs. 8(b) and 8(c) present the flow structures of modes 1 and 3 for $F r=0.23$ and 0.47 . These are similar to those identified with the POD analysis. The main difference occurs for $\Phi_{D M D 1}$ at $F r=0.47$, where the in-plane stagnation points identified by the POD mode [Fig. 5(a)] are not present in the corresponding DMD mode
[Fig. 8(b)]. This discrepancy could be explained by considering that DMD is capable of isolating structures with a specific frequency; therefore, it might be expected that higher order POD modes of lower kinetic energy content are characterised by a similar frequency. This discrepancy does not affect the main feature of the flow which, similar to the POD analysis, is denoted by two streams of opposite directions along the wall of the bioreactor.

\section{B. Lagrangian structures of the flow in OSBs}

The FTLE fields were calculated using all the three velocity components only for the lowest Froude number, $F r=0.23$, and $h / d_{i}=0.45$. First, PIV data measurements were carried out for different horizontal planes, $z / d_{i}=0.015-0.165$, at intervals of $\Delta z / d_{i}=0.015$. Second, 36 vertical PIV measurements were carried out from $\phi=0-175^{\circ}$ at intervals of $\Delta \phi=5^{\circ}$. Finally, by azimuthally stacking the vertical measurements and interpolating between the angles, the axial velocity component is obtained for every point in each horizontal plane measured. It should be noted that the horizontal velocity fields were computed using the LOM of the POD in Eq. (4), while the vertical fields were obtained by averaging 500 instantaneous measurement fields for each one of the 36 different phase angles. Figure 9(a) shows the FTLE fields computed at $z / d_{i}=0.03$ for $\phi=0^{\circ}$, i.e., at the time when the cylinder is at its most leftward position in its cycle. In order to reduce the influence of noise and the choice of phase angle, the FTLE fields were computed at every $\Delta \phi=5^{\circ}$ and averaged throughout the cycle, with the average field, $\overline{\sigma_{f}}$, shown in Fig. 9(b). Both the instantaneous and average FTLE fields are dominated by a circular ridge at $r / d_{i} \approx 0.4$. Closer to the axis, at $r / d_{i} \approx 0.2-0.3, \sigma_{f}$ is very low, and there is a region of moderate intensity near the centre at $r / d_{i} \lesssim 0.2$.

In order to identify Lagrangian coherent structures and divide the flow into qualitatively distinct regions, the variations in $\sigma_{f}$ and its relation to the overall flow are assessed. The two regions of moderate and elevated Lyapunov exponents at $r / d_{i} \lesssim 0.2$ and $\approx 0.4$ can be explained by considering the role of the central vortex in the vessel and the total displacement of tracers, respectively.

When considering the effect of the central vortex, it is important to distinguish between the vorticity due to the coherent vortex and that due to shear. One such method to do this is to use the swirling parameter, ${ }^{34}$ rather than the vorticity. The swirling parameter is an Eulerian metric, defined as

$$
\Lambda_{c, i}=\lambda_{c, i} \frac{\omega_{z}}{\left|\omega_{z}\right|},
$$

where $\lambda_{c, i}$ is the largest complex component of the eigenvalue of the velocity gradient tensor and $\omega_{z}$ is the vorticity (both of which are here calculated in the $z / d_{i}=0.03$ plane). The complex eigenvalue is zero outside of coherent vortices, even if considerable vorticity is present due to shear. The vorticity term in Eq. (11) ensures that the sign of the swirling parameter corresponds to the sign of the vortex at a given point.

As before, the swirling field is averaged throughout the cycle to reduce the influence of the choice of phase angle. The $\overline{\Lambda_{c, i}}$ field in Fig. 10(a) indicates that the region of flow occupied by a coherent vortex is relatively small. The light blue 

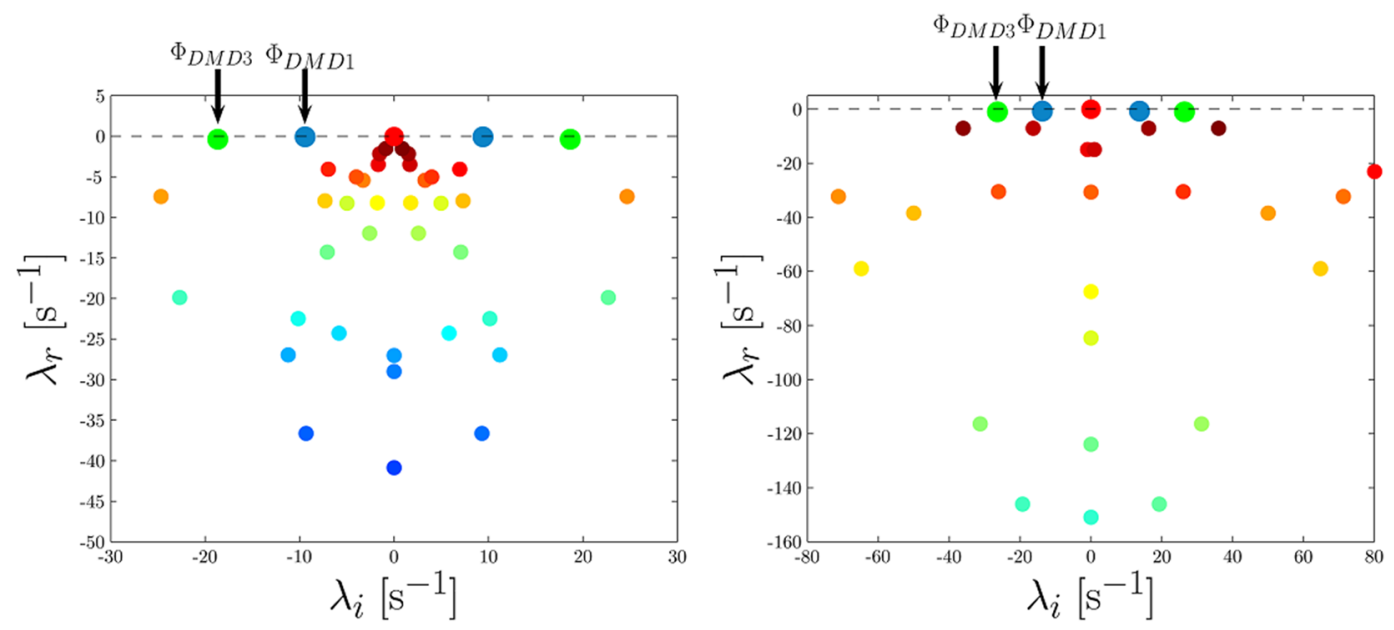

(a)
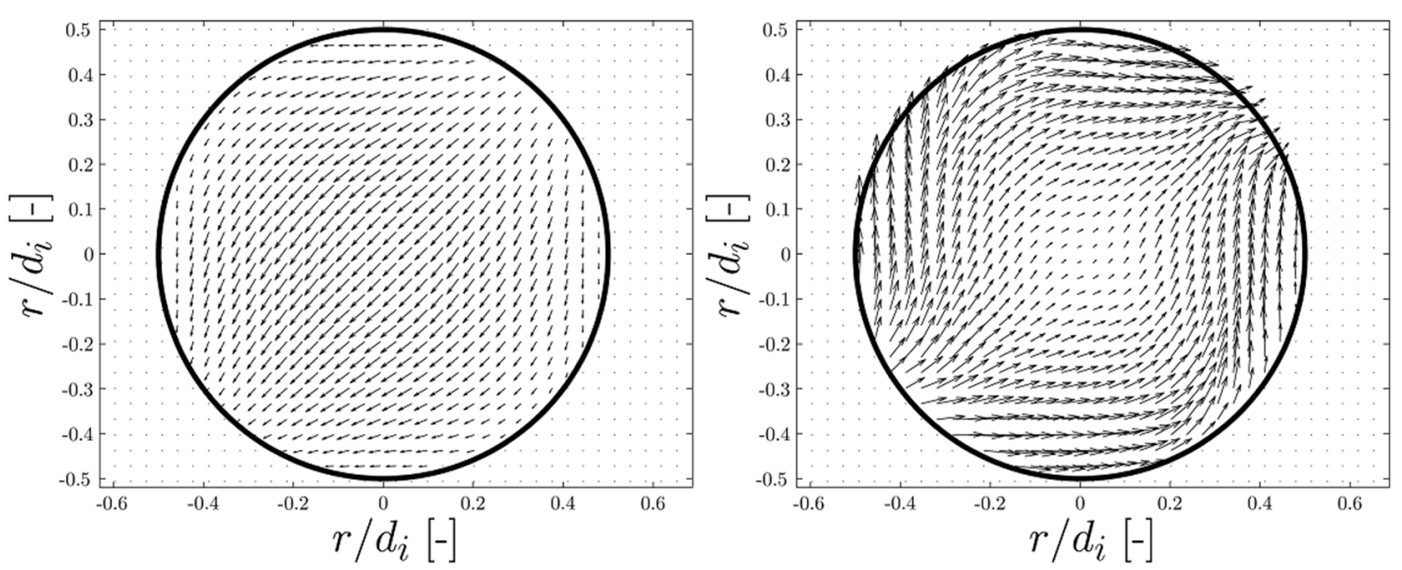

(b)
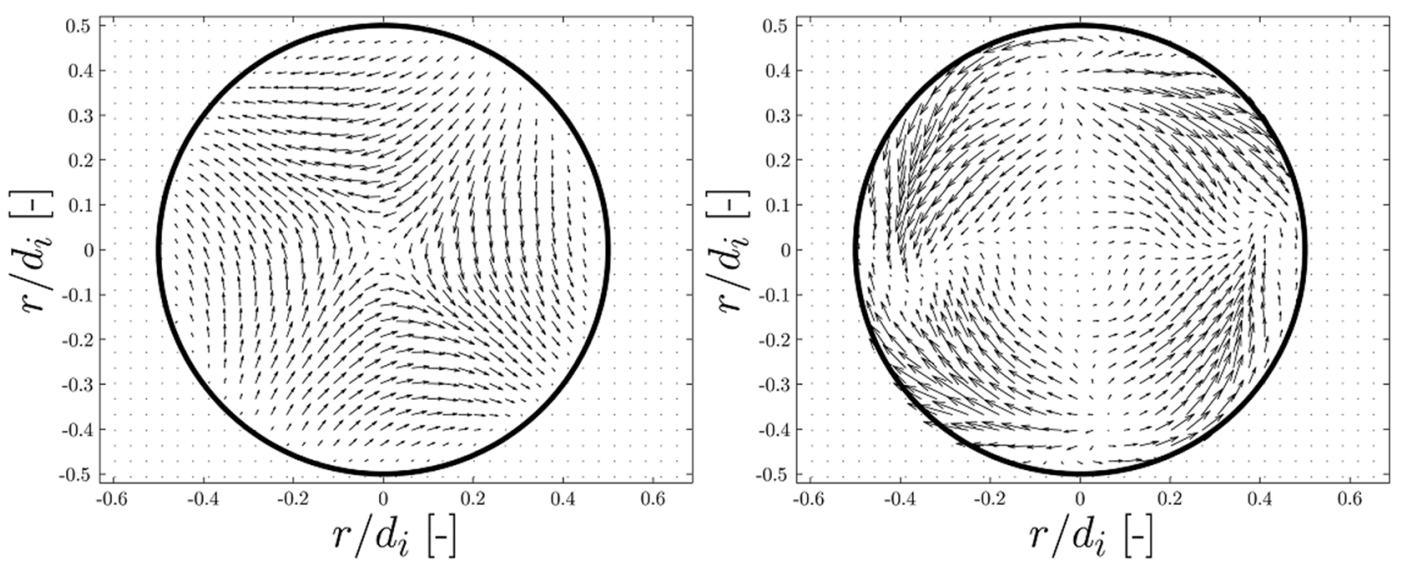

(c)

FIG. 8. (a) Eigenvalue spectrum associated with the different modes for $F r=0.23$ (left figure) and $F r=0.47$ (right plot); (b) velocity field of $\Phi_{D M D 1}$ for $F r=0.23$ (left plot) and $F r=0.47$ (right plot); (c) velocity field of $\Phi_{D M D 3}$ for $F r=0.23$ (left plot) and $F r=0.47$ (right plot) $\left(h / d_{i}=0.45\right.$ and $z / d_{i}=0.1$ ). The symbol size and colour of the eigenvalues are intended to separate large scale energetic structures (large symbols) from smaller-scale less energetic structures (small symbols).

line corresponds to $r / d_{i}=0.02$, where there exists a broad region of non-zero Lyapunov exponent (Fig. 9) and indicates that this regions of $\sigma_{f}$ is associated with the presence of a coherent vortex. Despite the orbital motion of the vessel, the region of $r / d_{i} \approx 0.2-0.3$ has almost zero swirling strength and does not contain significant amount of coherent vorticity, 

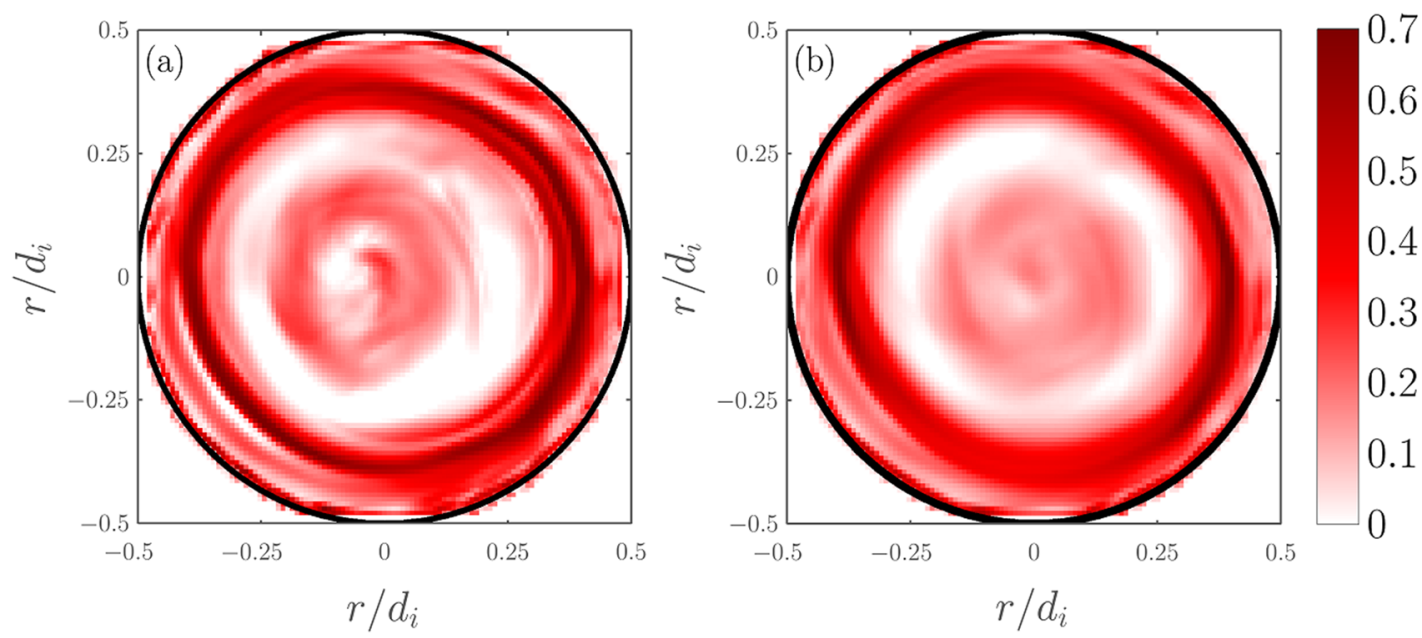

FIG. 9. (a) Instantaneous FTLE field at $z / d_{i}=0.03$, indicating regions of intense fluid stretching and the location of Lagrangian coherent structures, for $\phi=0^{\circ}$, and (b) the average of all corresponding forward FTLE fields computed at 72 equally spaced phases. The fields were computed over two shaking cycles and indicate that the fluid stretching occurs primarily near the walls $\left(r / d_{i} \approx 0.4\right)$ and in the centre of the vessel $\left(r / d_{i} \lesssim 0.2\right)$.

which explains the negligible Lyapunov exponent found in this region.

It seems reasonable to suggest that the other major feature in the $\sigma_{f}$ fields, the intense circular ridge at $r / d_{i} \approx 0.4$, is related to the boundary of the "wall region," where the motion of fluid is strongly affected by the viscous stresses surrounding the solid boundary. To test this hypothesis, the magnitude of the total three-dimensional displacement of tracers originating in the $z / d_{i}=0.03$ plane over two complete orbital cycles were calculated. The fields were computed using a start time at each phase, and the resulting displacement fields were averaged. The average displacement field, $\bar{s}$, is presented in Fig. 10(b), along with the location of the radial maximum of the average FTLE field at each phase angle (blue line). It is clear that the FTLE ridge separates regions where the total displacement of tracers is large $\left(\bar{s} / d_{i}>0.1\right)$ and regions near the wall where the displacement is small.

Fluid elements inside the wall region $\left(r / d_{i} \gtrsim 0.4\right)$ will experience relatively low rates of stretching due to their low overall displacement. By contrast, elements within the region closer to the axis $\left(r / d_{i} \approx 0.2-0.3\right)$ exhibit large displacements, but the absence of coherent vortices indicate that this motion does not result in significant deformation of the fluid elements. This may be thought of as fluid tracers moving "with" their neighbouring tracers, as occurs in a potential vortex.

The boundary between the wall and potential vortex regions, by contrast, is defined by intense stretching and high $\sigma_{f}$. This can be explained by considering two neighbouring tracers near this location, separated by a small radial distance. As the vessel is shaken, the outer tracer in the wall region displaces slowly, while the inner tracers in the potential vortex region displaces much faster, leading to their rapid separation and strongly local stretching. The presence of a maxima in $\sigma_{f}$ indicates that this region is a Lagrangian coherent structure and a repelling line. ${ }^{30}$

In this manner, the Lyapunov exponent, swirling parameter and displacement fields allows us to divide the flow into four distinct regions: (1) a coherent vortex with moderate stretching at the centre of the vessel $\left(r / d_{i} \lesssim 0.2\right)$;
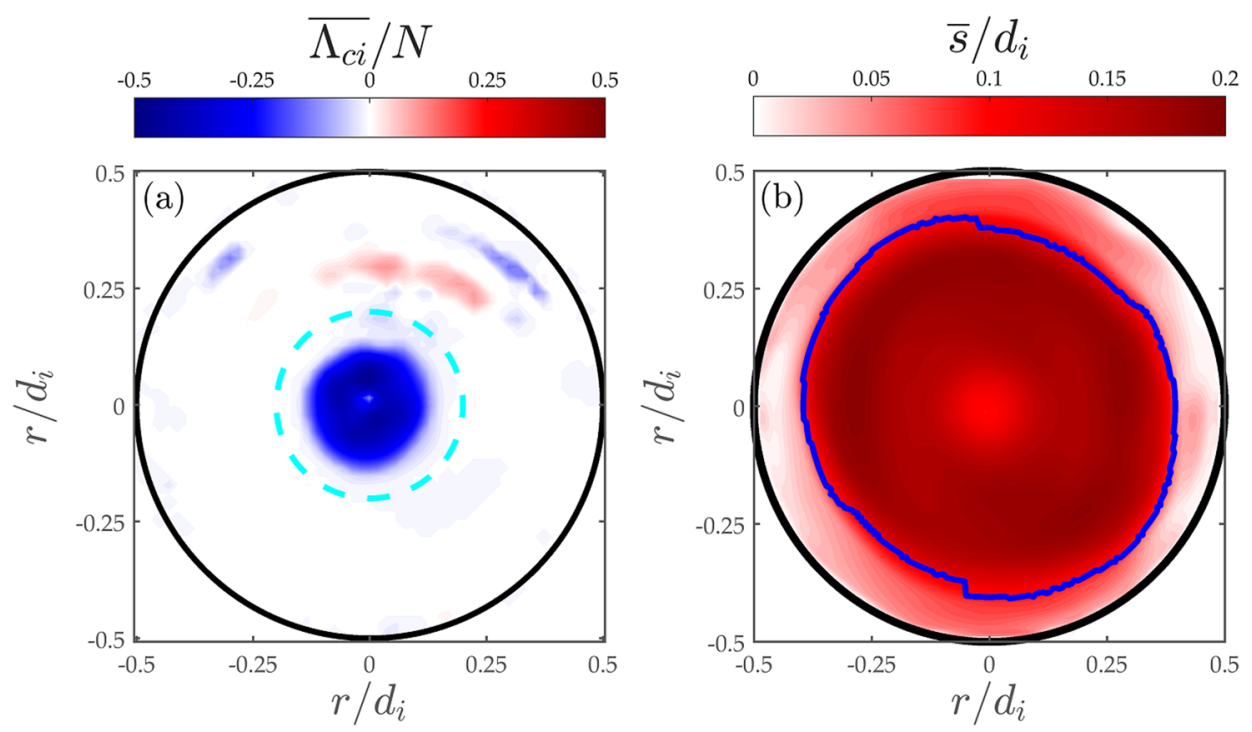

FIG. 10. (a) Swirling parameter, averaged over an entire shaking cycle, indicating the presence of a single large coherent vortex in the centre of the vessel. The cyan line at $r / d_{i}=0.2$ indicates the approximate location of the region of elevated FTLE (Fig. 9). (b) The average magnitude of total three-dimensional displacement of fluid tracers over two shaking cycles. The dark blue line shows the radial maximum in the FTLE fields at $r / d_{i} \approx 0.4$ (Fig. 9), and coincides with the boundary between the inner flow and the wall region. 
(2) a potential vortex region with negligible stretching $\left(r / d_{i} \approx 0.2-0.3\right)$; (3) a narrow band of strong stretching which acts as a repelling line $\left(r / d_{i} \approx 0.4\right)$; and (4) a wall region where fluid displacement is small $\left(r / d_{i} \gtrsim 0.4\right)$. These findings are in agreement with the mixing time reported by Rodriguez et al. ${ }^{19}$ who investigated how the radial coordinate of the feed insertion point affected the overall macro-mixing performances of the bioreactor. From the mixing maps they produced both for in-phase and out-of-phase conditions, it was evident that local mixing dynamics were faster closer to the wall by a factor of 2.5. Similarly, investigation on optimum feed location showed that when the reagent was injected in the vortex core, the overall mixing was approximately two folds longer than when insertion was made next to the wall. These results were more consistent at $F r=0.24$, when the axial vortex after flow transition is well defined and feed insertion inside and outside of the vortex is more systematic and repeatable.

\section{CONCLUSION}

This is the first study to provide insight on the flow in a shaken system by means of mode decompositions and Lagrange Coherent Structures (LCS) approaches. These techniques can offer an effective and alternative approach to standard phase-resolved and mixing time measurements to assess the dynamics in a shaken system. POD and DMD techniques were employed to characterise the flow for conditions before $(F r=0.23)$ and after flow transition $(F r=0.47)$. For both $F r$, modes 1 and 2 were associated with the rotational frequency of the shaker table and therefore to the main oscillatory motion of the free surface; however, their percentage energy content differed significantly. For $F r=0.23$, the first two modes contained $96 \%$ of the total kinetic energy and exhibited a strong central stream parallel to the reactor orbital velocity. The stream linked two in-plane stagnation points, where the flow is redirected vertically. These structures are in agreement with those observed in phase-resolved velocity measurements. ${ }^{12}$ When the Froude number was increased to $F r=0.47$, the first two modes displayed different spatial structures and contained only $80 \%$ of the total energy. In this case, the flow was locally forced to follow the bioreactor wall curvature, while in the core of the vessel, a region of low velocity was observed. The superimposition of the first two modes and the mean flow exhibited swirling characteristics, where the vessel side further away from the orbit centre exhibited greater velocity. For the highest $\mathrm{Fr}$ considered, the energy content of modes 3 and 4 become more significant, $\approx 10 \%$, and it corresponds to a frequency double that of the orbital motion. This could be linked to free surface shape variation already observed in the study of Reclari et al. ${ }^{16}$ The FTLE analysis was carried out for $F r=0.23$ and allowed us to distinguish four regions within the bioreactor: (1) a region in the centre of the vessel where moderate stretching occurs; (2) a region with negligible stretching located at $r / d_{i}=0.2-0.3$; (3) a circular ridge at $r / d_{i}=0.4$; and (4) a region near the wall where the fluid displacement is small at $r / d_{i} \gtrsim 0.4$. The analysis conducted in this investigation offer a novel prospective on the flow and mixing dynamics in shaken reactors and can initiate the development of an alternative approach to correlate flow structures in stirred and shaken technologies, potentially providing an effective tool to improve and bridge scaling strategies between the systems.

\section{ACKNOWLEDGMENTS}

Financial support for the work reported here was provided by the Engineering and Physical Sciences Research Council (EPSRC) of the UK, Grant No. EP/E055958.

${ }^{1}$ H. Zhang, S. Lamping, S. Pickering, G. Lye, and P. Shamlou, "Engineering characterisation of a single well from 24-well and 96-well microtitre plates," Biochem. Eng. J. 40, 138-149 (2008).

${ }^{2}$ W. Klöckner and J. Büchs, "Advances in shaking technologies," Trends Biotechnol. 30, 307-314 (2012).

${ }^{3}$ N. Raven, S. Rasche, C. Kuehn, T. Anderlei, W. Klöckner, F. Schuster, M. Henquet, D. Bosch, J. Büchs, R. Fischer, and S. Schillberg, "Scaledup manufacturing of recombinant antibodies produced by plant cells in a 200-L orbitally-shaken disposable bioreactor," Biotechnol. Bioeng. 112, 308-321 (2015).

${ }^{4}$ X. Zhang, M. Stettler, D. D. Sanctis, M. Perrone, N. Parolini, M. Discacciati, M. D. Jesus, D. Hacker, A. Quarteroni, and F. Wurm, "Use of orbital shaken disposable bioreactors for mammalian cell cultures from the milliliter-scale to the 1000-liter scale," Adv. Biochem. Eng./Biotechnol. 115, 33-53 (2010).

${ }^{5}$ C. Pena, C. Peter, J. Büchs, and E. Galindo, "Evolution of the specific power consumption and oxygen transfer rate in alginate-producing cultures of Azotobacter vinelandii conducted in shake flasks," Biochem. Eng. J. 36, 73-80 (2007).

${ }^{6}$ A. Lara, E. Galindo, O. Ramirez, and L. Palomares, "Living with heterogeneities in bioreactor," Mol. Biotechnol. 34, 355-381 (2006).

7 J. Gardner and G. Tatterson, "Characterization of mixing in shaker table containers," Biotechnol. Bioeng. 39, 794-797 (1992).

${ }^{8}$ J. Büchs, U. Maier, C. Milbradt, and B. Zoels, "Power consumption in shaking flasks on rotatory shaking machines: I. Power consumption measurement in unbaffled flasks at low liquid viscosity," Biotechnol. Bioeng. 68, 589-593 (2000).

${ }^{9}$ J. Büchs, S. Lotter, and C. Milbradt, "Out-of-phase operating conditions, a hitherto unknown phenomenon in shaking bioreactors," Biochem. Eng. J. 7, 135-141 (2001).

${ }^{10}$ H. Zhang, W. Dalson, E. Moore, and P. Shamlou, "Computational fluid dynamics (CFD) analysis of mixing and gas liquid mass transfer in shake flasks," Biotechnol. Appl. Biochem. 41, 1-8 (2005).

${ }^{11}$ H. Kim and J. P. Kizito, "Stirring free surface flows due to horizontal circulatory oscillation of partially filled container," Chem. Eng. Commun. 196(11), 1300-1321 (2009).

${ }^{12}$ W. Weheliye, M. Yianneskis, and A. Ducci, "On the fluid dynamics of shaken bioreactors-flow characterization and transition," AIChE J. 59, 334-344 (2013).

${ }^{13}$ A. Ducci and W. Weheliye, "Orbitally shaken bioreactors viscosity effects on flow characteristics," AIChE J. 60, 3951-3968 (2014).

${ }^{14}$ G. Rodriguez, I. Pieralisi, T. Anderlei, A. Ducci, and M. Micheletti, "Appraisal of fluid flow in a shaken bioreactor with conical bottom at different operating conditions," Chem. Eng. Res. Des. 108, 186-197 (2016).

${ }^{15}$ J. M. D. Thomas, A. Chakraborty, R. E. Berson, M. Shakeri, and M. K. Sharp, "Validation of a CFD model of an orbiting culture dish with PIV and analytical solutions," AIChE J. 63, 4233-4242 (2017).

${ }^{16}$ M. Reclari, M. Dreyer, S. Tissot, D. Obreschkow, F. M. Wurm, and M. Farhat, "Surface wave dynamics in orbital shaken cylindrical containers," Phys. Fluids 26, 052104 (2014).

${ }^{17}$ J. Bouvard, W. Herreman, and F. Moisy, "Mean mass transport in an orbitally shaken cylindrical container," Phys. Rev. Fluids 2, 084801 (2017).

${ }^{18}$ M. Discacciati, D. Hacker, A. Quarteroni, S. Quinodoz, S. Tissot, and F. Wurm, "Numerical simulation of orbitally shaken viscous fluids with free surface,” Int. J. Numer. Methods Fluids 71, 294-315 (2013).

${ }^{19}$ G. Rodriguez, W. Weheliye, T. Anderlei, M. Micheletti, M. Yianneskis, and A. Ducci, "Mixing time and kinetic energy measurements in a shaken cylindrical bioreactor," Chem. Eng. Res. Des. 91, 2084-2097 (2013).

${ }^{20}$ G. Rodriguez, T. Anderlei, M. Micheletti, M. Yianneskis, and A. Ducci, "On the measurement and scaling of mixing time in orbitally shaken bioreactors," Biochem. Eng. J. 82, 10-21 (2014).

${ }^{21}$ S. Tissot, M. Farhat, D. Hacker, T. Anderlei, and M. Kühner, "Determination of a scale-up factor from mixing time studies in orbitally shaken bioreactors," Biochem. Eng. J. 52, 181-186 (2010). 
${ }^{22}$ E. Olmos, K. Loubiere, C. Martin, G. Delaplace, and A. Marc, "Critical agitation for microcarrier suspension in orbital shaken bioreactors: Experimental study and dimensional analysis," Chem. Eng. Sci. 122, 545-554 (2015).

${ }^{23}$ I. Pieralisi, G. Rodriguez, M. Micheletti, A. Paglianti, and A. Ducci, "Microcarriers suspension and flow dynamics in orbitally shaken bioreactors," Chem. Eng. Res. Des. 108, 198-209 (2016).

${ }^{24}$ A. Ducci, Z. Doulgerakis, and M. Yianneskis, "Decomposition of flow structures in stirred reactors and implication for mixing enhancement," Ind. Eng. Chem. Res. 47, 3664-3676 (2008).

${ }^{25} \mathrm{Z}$. Doulgerakis, M. Yianneskis, and A. Ducci, "On the manifestation and nature of macro-instabilities in stirred vessels," AIChE J. 57, 2941-2954 (2011).

${ }^{26}$ A. Line, "Eigenvalue spectrum versus energy density spectrum in a mixing tank," Chem. Eng. Res. Des. 108, 13-22 (2016).

${ }^{27}$ G. Berkooz, P. Holmes, and J. Lumley, "The proper orthogonal decomposition in the analysis of turbulent flows," Annu. Rev. Fluid Mech. 25, 539-575 (1993).
${ }^{28}$ P. J. Schmid, "Dynamic mode decomposition of numerical and experimental data," J. Fluid Mech. 656, 5-28 (2010).

${ }^{29}$ S. L. Brunton, J. L. Proctor, J. H. Tu, and J. N. Kutz, "Compressed sensing and dynamic mode decomposition," J. Comput. Dyn. 2, 165-191 (2015).

${ }^{30}$ S. C. Shadden, F. Lekien, and J. E. Marsden, "Definition and properties of Lagrangian coherent structures from finite-time Lyapunov exponents in two-dimensional aperiodic flows," Phys. D 212, 271-304 (2005).

${ }^{31} \mathrm{~N}$. Cagney and S. Balabani, "Lagrangian structures and mixing in the wake of a streamwise oscillating cylinder," Phys. Fluids 28, 045107 (2016).

${ }^{32}$ M. Mathur, G. Haller, T. Peacock, J. E. Ruppert-Felsot, and H. L. Swinney, "Uncovering the Lagrangian skeleton of turbulence," Phys. Rev. Lett. 98, 144502 (2007).

${ }^{33}$ K. R. Pratt, J. D. Meiss, and J. P. Crimaldi, "Reaction enhancement of initially distant scalars by Lagrangian coherent structures," Phys. Fluids 27, 035106 (2015).

${ }^{34} \mathrm{Y}$. Wu and K. T. Christensen, "Population trends of spanwise vortices in wall turbulence," J. Fluid Mech. 568, 55-76 (2006). 